\title{
Artículo Original \\ Caracterización química de los extractos colorantes de siete especies forestales y del fijador natural, utilizado en 19 comunidades indígenas de Ucayali, Perú
}

\author{
[Chemical characterization of dyeing compounds found in seven timber \\ species, and of the natural fixer, used by 19 indigenous communities of \\ Ucayali, Peru]
}

\author{
Luisa Riveros $^{1 *}$, Lylyams I. Inga ${ }^{2}$ \\ ${ }^{1}$ Departamento de Ingeniería Agroforestal Acuícola, Universidad Nacional Intercultural de la Amazonía, Km. 0,5 Carretera \\ a San José de Tushmo, Yarinacocha, Perú. \\ 2Departmento de Ciencias, Universidad Nacional Intercultural de la Amazonía, Km. 0,5 Carretera a San José de Tushmo, \\ Yarinacocha, Perú. \\ e-mail: riverosluisa@yahoo.com
}

\begin{abstract}
Resumen
Se realizó la caracterización química de las sustancias colorantes provenientes de las 07 especies y del fijador natural identificadas en la primera parte de la investigación, y que son utilizados como materia prima para el teñido de telas y artesanías por 19 comunidades indígenas de la Región Ucayali. Estas especies son: Trichilia maynasiana, Trichilia poeppigiana, Swietenia macrophylla, Buchenavia parvifolia, Terminalia oblonga, Terminalia sp., y Picramnia juniniana. Los tintes y cortezas de estas especies sometidas a un tamizaje fitoquímico presentaron mayormente taninos, lactonas y flavonoides, en diferentes concentraciones, como principales metabolitos, mientras que la especie Picramia juniniana "ami", de coloración lila, presento básicamente quinonas. En la arcilla empleada como fijador, denominada greda, barro especial o "mano", se encontraron 32 elementos químicos con predominancia de silicio y aluminio con un $\mathrm{pH}$ ácido, mientras que el fijador reacciona con el tinte formando complejos denominados quelatos los cuales fijan el color sobre las fibras naturales.
\end{abstract}

Palabras clave: Colorantes naturales, composición química, uso indígena, Ucayali, Perú.

\begin{abstract}
The chemical characterization of dyeing compounds extracted from 07 species and of the natural fixer identified in the first part of the investigation and used by 19 native communities of the Region Ucayali was carried out.These species are: Trichilia maynasiana, Trichilia poeppigiana, Swietenia macrophylla, Buchenavia parvifolia, Terminalia oblonga, Terminalia sp., and Picramnia juniniana. After a phytochemical screening, the dyes and bark pieces of the species analysed, presented tannins, lactones and flavonoids at different concentrations as the main metabolites. Only the Picramnia juniniana "ami" species used for lilac color, presented mainly quinones. The chemical composition of the fixer clay or "mano", presented 32 chemical elements with predominance of silice and aluminium with acid $\mathrm{pH}$, while the fixer reacts with the dye forming chelates, which fix the color in the natural fabrics.
\end{abstract}

Keywords: Natural coloration, chemistry composition, indegenous use, Ucayali, Perú. 


\section{INTRODUCCIÓN}

Los bosques húmedos tropicales, proporcionan diversos beneficios maderables y no maderables que las comunidades indígenas de la etnia Shipibo-Conibo del Departamento de Ucayali, conocen y han utilizado ancestralmente. Según Cajías (2006),actualmente en el mundo se está retomando el interés por usar colorantes naturales en reemplazo de los sintéticos, que han resultado ser tóxicos cuando se emplean en alimentos, conservas, tintes para textiles, etc. El arte del teñido parece fácil, pero implica muchos factores que a simple vista pasan desapercibidos, como el colorante "natural" a aplicar, el fijador o mordiente, que en este caso es la "greda o barro blanco", y el tipo de tela "algodón", usado por las comunidades indígenas.

Los tintes naturales en las comunidades indígenas de Ucayali, se extraen de las cortezas de las plantas, las que luego son cortadas y colocadas en agua caliente hasta que el color sea transferido al agua y cuando se le añade un mordiente, el tinte se adhiere a la fibra del material. INFOMIPYME (2008), indica que los tintes naturales tienen un valor incalculable para la elaboración de las artesanías en los diversos pueblos amazónicos, porque pueden usarse en el teñido de hilo, algodón, lanas, telas, yute, palma, cuero, pieles, plumas, paja, madera, flores naturales y artificiales, aserrín entre otros También para la población indígena, los colores, eran manifestación simbólica de lo sagrado y al mismo tiempo, de desigualdades sociales, dominación, poder político, económico y guerrero, y los diversos colores de hilos tenían su significado.

Hay una gran variedad de plantas que se pueden utilizar para hacer tintes vegetales $y$, que existen y se pueden obtener en casi todo el mundo, sin embargo, cada zona tiene sus propias plantas con sus propios pigmentos y que pueden usar para hacer tintes. Existen dos tipos primarios de pigmentos naturales utilizados para teñir, los pigmentos liposolubles y los hidrosolubles. Entre los liposolubles tenemos la clorofila o los carotenoides, que están en todas las plantas, y que producen color verde a verde oliva y amarillos a rojos respectivamente, mientras que en los hidrosolubles existen los flavonoides como el rosado-púrpura que se encuentra en la remolacha y el amarillo en la cáscara de la cebolla.

Arango (2008), afirma que cuando el color es añadido a un material saturado en mordiente, el tinte se adhiere a la fibra del material y los mordientes ayudan a que los colores se adhieran permanentemente a las fibras, existen muchos mordientes que son muy tóxicos, por utilizar sulfato de amonio o de aluminio, comúnmente utilizado como un agente de curtiembre. Los nativo-americanos utilizaban un gran número de mordientes que se dan en la naturaleza como el alumbre natural que se precipita sobre algunos suelos, ácidos tánicos de zumaque (bayas, ramas u hojas), detergente natural a base de cenizas de madera, orina, mezcla de excrementos de oveja con agua y humo.

El término "mordiente", según Pimentel (2008), proviene del verbo latino "mordere" que significa morder en el sentido de asir o fijarse en una cosa o reforzar su acción. Es importante en el proceso de teñido, ya que sin el mordiente adecuado los colores ofrecen un aspecto pobre y heterogéneo. Se emplean diversas sustancias como mordiente; algunas son totalmente seguras, pero otras requieren un manejo cuidadoso. El tipo de tinte $y$ mordiente a escoger dependerá del color, tono y la clase de fibra que se va a emplear, pudiéndose obtener distintos colores y tonos, en función de la sustancia utilizada como mordiente.

Cajías (2006) señala, que la acidez o alcalinidad de un tinte afecta el resultado del teñido. Por ello es importante controlar el $\mathrm{pH}$, ayudando a que los colores sean más firmes y resistentes a la luz solar, y los mordientes pueden modificar los colores, en algunos casos dándoles más brillo o viveza, en otros oscureciéndolos, y en otros transformando el color original en uno nuevo. El alumbre, el amoníaco y el crémor tártaro dan más brillo y logran colores más vivos. El hierro tiende a oscurecer los colores. Los taninos dan un tono más profundo a colores como el gris y el café y funciona mejor con fibras vegetales (algodón, yute, etc.), produciendo colores profundos, resistentes a la luz solar, con colores suaves y naturales a diferencia de los tintes sintéticos.

En líneas generales, se conoce la interacción que hay entre el tinte y el fijador, pero 
específicamente en las especies vegetales con sustancias de coloración utilizadas en la selva amazónica no se tiene información por lo que el propósito del presente estudio fue contribuir en el conocimiento de los componentes químicos que tienen las especies forestales y no forestales así como del mordiente que son utilizadas como materia prima para elaborar sus artesanías en telas por las comunidades indígenas de Ucayali, ya que nada de información se tiene sobre las características químicas de estas especies que son utilizadas ancestralmente y que con el pasar tiempo se puede perder, debido a que este conocimiento se transmite de padres a hijos en forma verbal, siendo el objetivo determinar la caracterización química de las especies tintóreas y del fijador que es utilizado por las comunidades indígenas shipibo-conibo de la Región Ucayali.

\section{MATERIALES Y MÉTODOS}

El presente estudio consistió en visitar las comunidades indígenas Cacataibo y ShipiboConibo de la Región Ucayali: Santa Rosa, Mebanañu, San Francisco, Santa Clara, Nuevo Egipto, Santa Teresita de Cashibo-Cocha, Nuevo San Juan, Puerto Firmeza, San Salvador, Callería, Patria Nueva, Saposoa, Puerto Belén, Roya, Utucuro, Nueva Betania, Palestina, Bethel y Santa Rosita de Abujao, para la búsqueda de información y toma de muestras de las especies con tintes y mordientes que utilizan en sus teñidos. Luego las muestras fueron enviadas a laboratorios para los análisis respectivos y determinar su caracterización química.

Para el análisis fitoquímico se utilizaron tintes extraídos por los artesanos y cortezas con sustancias de coloración, de las especies Swietenia macrophylla "caoba", Trichilia maynasiana, Trichilia poeppigiana, "Uchumullaca", Terminalia oblonga, Terminalia sp., Buchenavia parvifolia, y Picramnia juniniana. El tamizaje fitoquímico, se realizó con dos tipos de muestras, uno como extracto líquido y el otro a partir de cortezas (Figura 1), en un total de 11 muestras. Del grupo de muestras liquidas de tintes se extrajeron $250 \mathrm{ml}$ que se colocó en el liofilizador, obteniéndose un extracto liofilizado con peso aproximado de 5 a $6 \mathrm{~g}$. para realizar las pruebas respectivas; mientras que las muestras de cortezas se molieron, utilizando $200 \mathrm{~g}$. de muestra seca de los géneros Swietenia, Trichilia y Bouchenavia que se secaron a temperatura controlada, que no sobrepasó los $35^{\circ} \mathrm{C}$, luego fueron molidas hasta una granulometría correspondiente a un tamiz no 08 , después del cual se procedió a la maceración con etanol absoluto por 48 horas, llevándose al rotavapor a presión reducida obteniéndose de los géneros Sweitenia, Trichilia y Bouchenavia, un extracto etanólico de $8,76 \mathrm{~g}, 1,60 \mathrm{~g}$ y $1,33 \mathrm{~g}$, y un rendimiento de $4,38 \%, 0,80 \%$ y $0,66 \%$ respectivamente. Los extractos liofilizados y etanólicos fueron disueltos en ultrasonicador, y se procedió a realizar la evaluación fitoquímica, identificando los metabolitos secundarios, por medio de las reacciones específicas para cada metabolito según el método Matos 1998, Lock 1994 y Schabra et al. 1984.

En el caso de la preparación del mordiente (fijador), los artesanos de Ucayali diluyen la arcilla "mano" (en dialecto shipibo) en agua fría y la aplican directamente sobre el dibujo o franja que desean oscurecer, dejando secar la arcilla para que se impregne en el tejido y luego enjuagan la tela (Figura 2). Para la determinación de los componentes químicos de la arcilla, se envió las muestras al laboratorio de suelos del Instituto Nacional de Investigación Agraria (INIA) y al Laboratorio de Química de la Pontificia Universidad Católica del Perú (PUCP). Según el informe del INIA 2008, se utilizó diferentes técnicas para corroborar los componentes químicos, utilizando para el $\mathrm{pH}$ un potenciómetro con una relación de suelo/agua: $(1: 2,5)$; para cuantificar $\mathrm{K}, \mathrm{P}$, se utilizó el reactivo $\mathrm{NaHCO}-$ EDTA-SUPERFLOC, en el caso de Ca y $\mathrm{Mg}$ fue por extracción con $\mathrm{KCL}$, y para $\mathrm{K}$, Ca y $\mathrm{Mg}$ por absorción atómica, los métodos analíticos utilizados por el INIA fueron de los autores Ayre y Román, 1992. En la PUCP el método fue el propio del laboratorio: LAQ IAR01PUCP, que consistió en cuantificar los metales presentes en la muestra de barro por medio del equipo de absorción atómica y los resultados se expresaron en base seca, para lo cual previamente se determinó la humedad de la muestra (PUCP 2009). 

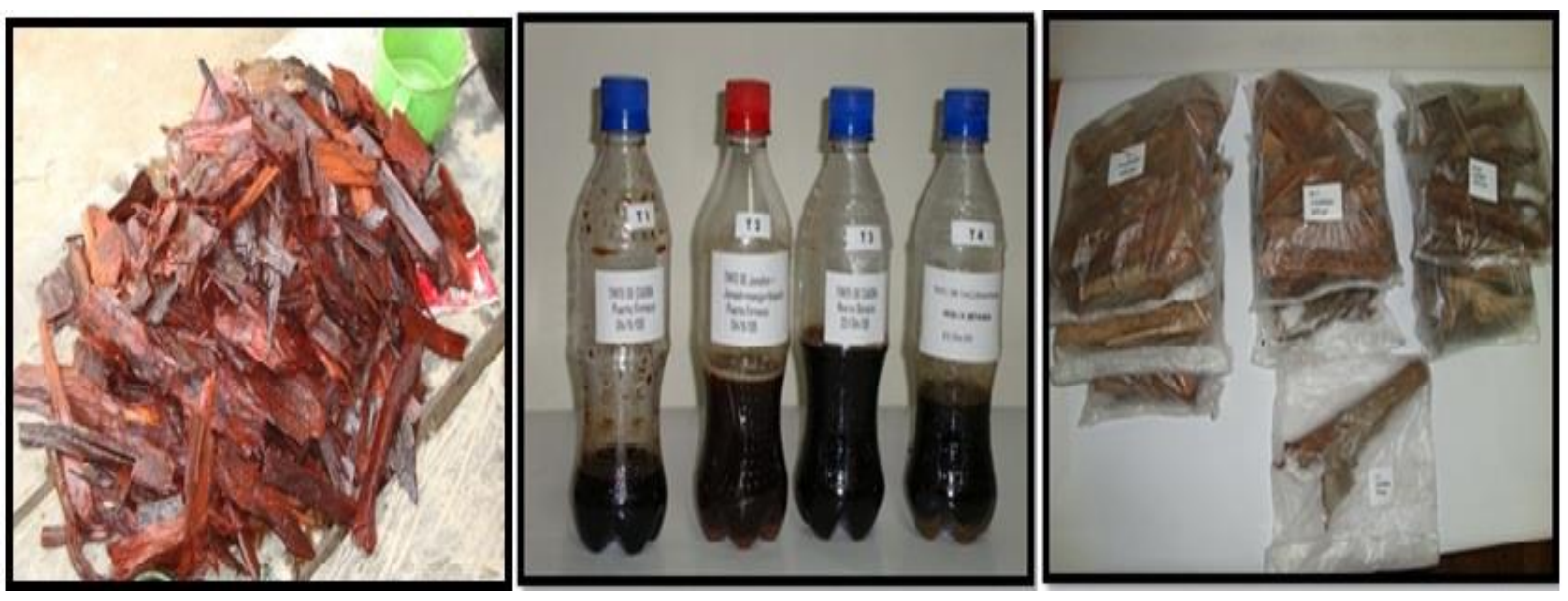

Figura 1. Cortezas con propiedad tintórea, muestras de corteza y tinte natural.
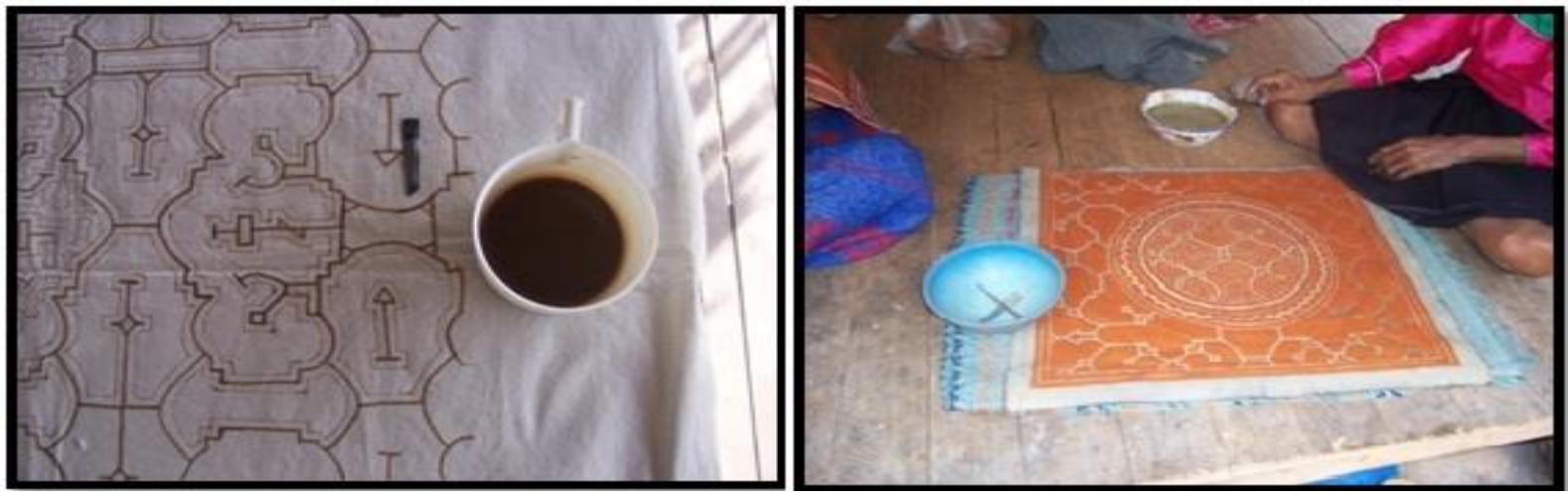

Figura2. Tinte extraído de las cortezas y fijación del color con mordiente "arcilla" llamado "mano" en shipibo.

\section{RESULTADOS Y DISCUSIÓN}

Los resultados que se encontró en las comunidades fueron que siguen dos pasos. Primero la extracción del tinte a partir de diversas cortezas (Figura 1) y luego la fijación del color en la superficie de la tela con una arcilla (mordiente) que extraen de las orillas de los meandros de los ríos. La preparación de tintes la realizan de forma artesanal hirviendo las cortezas con agua, en ollas sobre leña, y reduciendo el volumen hasta casi $1 / 3$ del envase, luego son almacenadas $y$ guardadas por largas temporadas.
En cuanto a la composición química de las especies, se determinó (Cuadro 1), que presentan básicamente taninos, lactonas $\mathrm{y}$ flavonoides en diferentes concentraciones, excepto en Picramnia juniniana (ami), que presenta solamente quinonas (UNAP, 2010); estos datos tienen relación con lo publicado por Abadía et al., 2003, mencionado por Pino et al, 2003, que afirman que los mejores resultados se obtienen con las hojas (29\%), tallo $(21 \%)$, semillas y frutos $(18 \%)$. 
Cuadro 1. Elementos fitoquímicos determinados en la corteza y en el extracto líquido de las especies con sustancia de coloración.

\begin{tabular}{|c|c|c|c|c|c|c|c|}
\hline No. & $\begin{array}{c}\text { Elementos } \\
\text { fitoquímicos }\end{array}$ & $\begin{array}{c}\text { Terminalia } \\
\text { sp. }\end{array}$ & $\begin{array}{c}\text { Terminalia } \\
\text { oblonga }\end{array}$ & $\begin{array}{l}\text { Trichilia } \\
\text { sp. }\end{array}$ & $\begin{array}{c}\text { Buchenavia } \\
\text { parvifolia }\end{array}$ & $\begin{array}{c}\text { Swetenia } \\
\text { macrophylla }\end{array}$ & $\begin{array}{l}\text { Pricramnia } \\
\text { juniniana }\end{array}$ \\
\hline 1 & Alcaloides & + & - & - & + & - & - \\
\hline 2 & Triterpenos & - & - & - & - & - & - \\
\hline 3 & Esteroides & - & - & + & - & - & - \\
\hline 4 & Quinonas & - & - & - & - & - & +++ \\
\hline 5 & Flavonoides & ++ & ++ & ++ & ++ & +++ & + \\
\hline 6 & Lactonas & +++ & +++ & +++ & +++ & +++ & - \\
\hline 7 & Saponinas & - & - & - & - & - & - \\
\hline 8 & $\begin{array}{l}\text { Aminas y } \\
\text { aminoácidos }\end{array}$ & - & - & - & - & - & - \\
\hline 9 & Fenoles & - & - & - & - & - & - \\
\hline 10 & Taninos & +++ & +++ & +++ & +++ & ++ & + \\
\hline
\end{tabular}

En cuanto al fijador, barro, greda, o "mano", de color blanquecino, según el análisis realizado en el INIA (2008), se determinó un alto porcentaje de silicio y aluminio, al igual que otros metales, como magnesio, calcio, potasio, y un $\mathrm{pH}$ ácido, debido a la presencia de aluminio; la muestra contenía también fracciones de limo, arena y materia orgánica. En la segunda muestra del fijador 0 arcilla el Laboratorio de la PUCP (2009), determinó que en su composición hay 32 elementos químicos presentes (Cuadro 2), y un alto porcentaje de silicio total de $30.13 \%$, de aluminio con $0,81 \%$, de hierro $0,21 \%$, de potasio $0,11 \%$, entre otros. El silicio es la base de la arcilla, dentro del cual se destacan otros metales como el hierro, potasio, calcio, magnesio y otros menores en trazas (ppm).

La greda, por el tipo de elementos metálicos que contiene, puede ser blanca o ligeramente oscura, de textura suave, característica de estas arcillas. La unión entre el tinte natural y la fibra se da por la interacción del mordiente (greda), que tiene afinidad por ellos y que al combinarse forma un precipitado insoluble sobre la fibra fijándola a esta. Esto ocurre porque la greda modifica la fibra, rompiendo sus enlaces moleculares, de modo que las moléculas del colorante natural puedan incorporarse, formando nuevos enlaces con carácter irreversible. 
Cuadro 2. Elementos químicos encontrados en las muestras de arcilla (fijador natural)

\begin{tabular}{|c|c|c|c|}
\hline \multirow[b]{2}{*}{ No. } & \multirow[b]{2}{*}{ Elementos químicos } & \multicolumn{2}{|c|}{ Resultados } \\
\hline & & Muestra 1 & Muestra 2 \\
\hline 1 & Aluminio total (\%) & 0,81 & 0,65 \\
\hline 2 & Arsénico total (ppm) & $<0,002$ & $<0,002$ \\
\hline 3 & Boro total (ppm) & $<0,03$ & 3,02 \\
\hline 4 & Bario total (ppm) & 46,20 & 46,98 \\
\hline 5 & Berilio total (ppm) & $<0,002$ & $<0,002$ \\
\hline 6 & Bismuto total (ppm) & $<0,20$ & $<0,20$ \\
\hline 7 & Calcio total (\%) & 0,07 & 0,09 \\
\hline 8 & Cadmio total (ppm) & $<0,006$ & $<0,006$ \\
\hline 9 & Cobalto total (ppm) & 1,20 & 1,81 \\
\hline 10 & Cromo total (ppm) & 4,10 & 4,54 \\
\hline 11 & Cobre total (ppm) & 5,80 & 18,35 \\
\hline 12 & Hierro total (\%) & 0,21 & 0,24 \\
\hline 13 & Potasio total (\%) & 0,11 & 0,16 \\
\hline 14 & Litio total (ppm) & $<0,04$ & $<0,04$ \\
\hline 15 & Magnesio total (\%) & 0,05 & 0,05 \\
\hline 16 & Manganeso total (ppm) & 30,90 & 32,86 \\
\hline 17 & Molibdeno total (ppm) & $<0,02$ & $<0,02$ \\
\hline 18 & Sodio total $(\%)$ & 0,03 & 0,05 \\
\hline 19 & Níquel total (ppm) & 4,30 & 8,67 \\
\hline 20 & Fósforo total (ppm) & 40,0 & $<0,3$ \\
\hline 21 & Plomo total (ppm) & 7,60 & 8,77 \\
\hline 22 & Antimonio total (ppm) & $<0,01$ & $<0,01$ \\
\hline 23 & Selenio total (ppm) & $<0,002$ & $<0,002$ \\
\hline 24 & Silicio total $(\%)$ & 25,15 & 30,13 \\
\hline 25 & Estaño total (ppm) & $<0,08$ & $<0,08$ \\
\hline 26 & Estroncio total (ppm) & 16,80 & 17,94 \\
\hline 27 & Titanio total (\%) & 0,03 & 0,04 \\
\hline 28 & Talio total (ppm) & $<0,04$ & $<0,04$ \\
\hline 29 & Vanadio total (ppm) & 8,50 & 8,67 \\
\hline 30 & Zinc total (ppm) & 21,50 & 29,64 \\
\hline 31 & Plata total (ppm) & $<0,01$ & 1,41 \\
\hline 32 & Mercurio total (ppm) & $<0,0002$ & $<0,0002$ \\
\hline
\end{tabular}


Entonces existe una estrecha relación entre el colorante vegetal, la greda o mordiente y el tipo de fibra en el que se aplicará el colorante vegetal, esta relación es originada por la quelatación que es la facilidad del compuesto químico (agente quelatante), para formar una estructura en anillo con un ión metálico, conteniendo dos o más ligandos potenciales lo que conduce a una buena interacción entre el colorante vegetal y la tela. La greda, facilita la formación de complejos coloreados, estabilizando el color en la superficie de la tela, debido a la abundante cantidad de metales que contiene, como silicio, aluminio, hierro, entre otros, los cuales interaccionan con los grupos cromóforos del colorante vegetal y por su abundancia son las sales de aluminio las que suelen dar los mejores resultados y los colores más vivos.

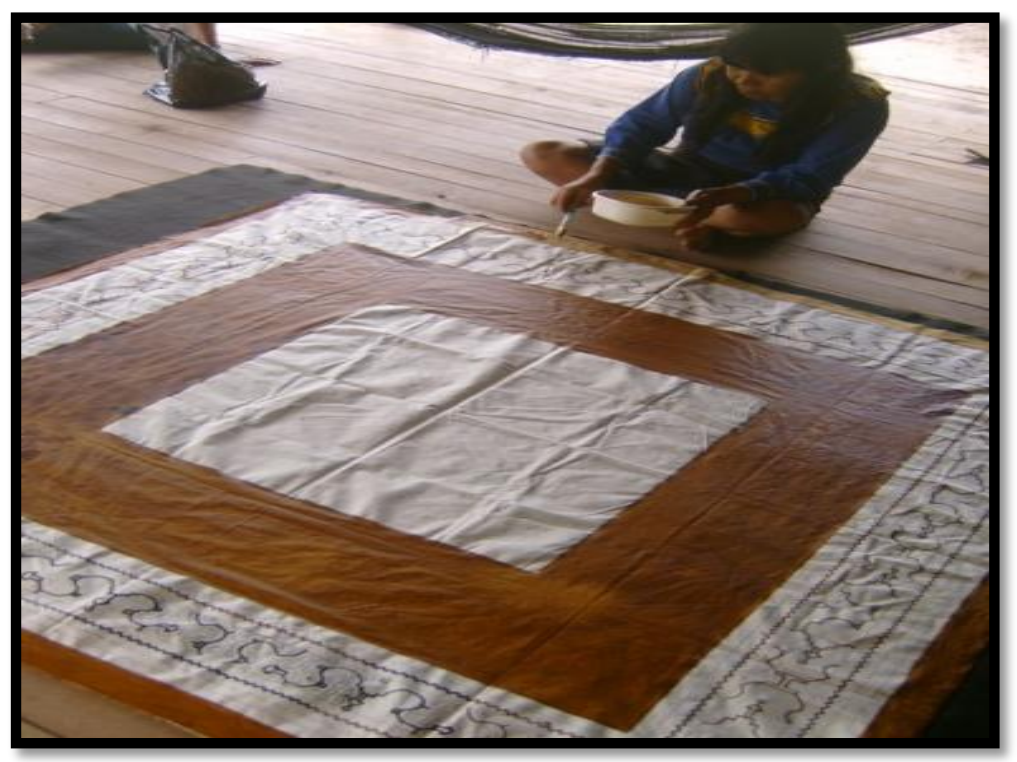

Figura 3. Teñido y dibujos con tintes naturales

\section{CONCLUSIONES}

Los componentes químicos más importantes y presentes en las especies estudiadas, son: taninos, lactonas, flavonoides, y quinonas.

El barro especial, arcilla o "mano" en shipibo, utilizado como mordiente o fijador del tinte natural, presentó como principales componentes químicos, el silicio y aluminio, que se encuentran en forma de sales.

El mordiente o fijador, presento 32 elementos químicos que forman parte de la estructura de los silicatos, que están en forma de sales. El pH del fijador es ácido por la presencia de aluminio.

\section{BIBLIOGRAFÍA}

Arango L. Taller de tintes naturales para lana. Artesanías de Colombia.

http://www.lablaa.org/blaavirtual/todaslasa rtes/tatinnapala/bibliografia.htm [Consultado 30 Mayo 2008].

Ayre O, Roman R. 1992. Métodos analíticos para suelos y tejido vegetal usados en el trópico húmedo. Lima, Perú.

Cajías M. 2006. Colores perdurables, colores efímeros. Los mordientes o fijadores en el proceso de teñido con tintes naturales. http://www.artesaniasdecolombia. com.co/documentos/documentos_pub/pcaji as.htm [Consultado 30 Julio 2008].

INFOMIPYME. Manual de tintes naturales. http://infomipyme.com/Docs/GT/Offline/tin tes.htm

[Consultado 18 Junio 2008]. 
INIA. 2008. Solicitud de Ensayo: SU0010 EEAP - 08. Laboratorio de suelos. Estación Experimental Agraria Pucallpa. Instituto Nacional de Investigación Agraria.

Lock O. 1994. Investigación Fitoquímica. Pontificia Universidad Católica del Perú, Lima.

Matos JA. 1998. Introducción a la fotoquímica experimental. Editorial UFC. Fortaleza, Brasil.

Pimentel J. Extracción de pigmentos y Tinción de tejidos.

http://www.jpimentel.com/ciencias_experi mentales/pagwebciencias/pagweb/la_cienci a_a_tu_alcance/Experiencias_biologia_tinte s_naturales.htm [Consultado 30 Mayo 2008].

Pino CW, Guerrero JE, Castro RA, Castro AA, Palacios JA y Castro A. 2003. Extracción artesanal de colorantes naturales, una alternativa de aprovechamiento de la diversidad biológica del Chocó, Colombia. Acta Biol Colomb 8 (2): 95-98.

PUCP. 2009. Informe de ensayo No 0355/2009- Laboratorio de análisis químicos- Sección Química-Departamento de Ciencias- Pontificia Universidad Católica del Perú, Lima.

Schabra SC, Ulso FC, Mshin E.N. 1984. Phytochemical Screening of Tanzanian Medical Plantas. J Etnopharmacol 11: 157159.

UNAP. 2010. Informe de evaluación fitoquímica de especies vegetales. Laboratorio de Investigación de Productos Naturales Antiparasitarios de la Amazonía (LIPNAA), Universidad Nacional de la Amazonía Peruana, Iquitos-Perú. 